Quim. Nova, Vol. 28, No. 2, 340-344, 2005

\title{
MAGNETO-V1: UM PROGRAMA PARA O CÁLCULO DE CORREÇÕES DIAMAGNÉTICAS E DE MOMENTOS MAGNÉTICOS EFETIVOS
}

\author{
Geraldo Roberto Friedermann, Giovana Gioppo Nunes e Jaísa Fernandes Soares* \\ Departamento de Química, Universidade Federal do Paraná, CP 19081, 81531-990 Curitiba-PR
}

Recebido em 16/6/04; aceito em 30/8/04; publicado na web em 12/11/04

\begin{abstract}
MAGNETO-V1: A PROGRAM FOR THE CALCULATION OF DIAMAGNETIC CORRECTIONS AND EFFECTIVE MAGNETIC MOMENTS. A new computer program has been developed to help the users of force methods for magnetic moment determination. It provides a user-friendly interface for the calculation of corrected magnetic susceptibilities of paramagnetic materials and enables the user to simulate a number of chemical formulations for the sample under study. The program is written in the Perl scripting language and runs on a Unix platform. A web interface for immediate use is freely available at http:// www.quimica.ufpr.br/cgi-bin/mag/mag.cgi.
\end{abstract}

Keywords: magnetic moment calculations; Gouy method; diamagnetic corrections.

\section{INTRODUÇÃO}

Historicamente, a susceptibilidade magnética $\chi$ foi uma das primeiras propriedades empregadas amplamente na caracterização de compostos de coordenação, atendendo a diversos objetivos. No ensino da Química, por exemplo, a medida da susceptibilidade magnética molar e do momento magnético efetivo permite ilustrar conceitos de estrutura eletrônica de forma simples e acessível experimentalmente ${ }^{1}$. Já na pesquisa científica, ela pode fornecer informação fundamental sobre a existência de ligações químicas, a nuclearidade de compostos de coordenação e a ocorrência de interações de troca entre centros magnéticos ${ }^{2}$. O valor do momento magnético efetivo, por sua vez, mesmo quando determinado apenas à temperatura ambiente, pode indicar o número de elétrons desemparelhados na espécie paramagnética, evidenciar estados de spin e informar sobre a dimensão do desdobramento de campo ligante em complexos ${ }^{3}$. O conhecimento das propriedades magnéticas de materiais, tais como anisotropia, permeabilidade, magnetização, remanência e histerese ${ }^{4}$, e de fenômenos magnéticos, como ferromagnetismo, superparamagnetismo, fotomagnetismo e nanomagnetismo ${ }^{5,6}$, entre outros, tem contribuído para o desenvolvimento científico e tecnológico em áreas tão diversas quanto biociências, catálise, química de coordenação e de materiais magnéticos ${ }^{4-7}$. Há ainda uma ligação forte entre o estudo do magnetismo molecular, a química sintética e a química teórica, uma vez que o "design" de compostos com um determinado comportamento magnético depende do entendimento dos mecanismos teóricos dos fenômenos magnéticos ${ }^{8,9}$.

Desde o tempo de Faraday, sabe-se que todas as substâncias são afetadas, em maior ou menor grau, por campos magnéticos externos. Um material diamagnético colocado num campo magnético não-homogêneo tende a ser repelido da região de campo mais forte, enquanto um material paramagnético, nas mesmas condições, tende a ser atraído. Nos métodos de Gouy e de Faraday para a determinação de susceptibilidades magnéticas, esta atração manifesta-se no aumento aparente de massa da amostra paramagnética na presença do campo ${ }^{10}$. No entanto, uma vez que todo material, mesmo paramagnético, possui um diamagnetismo intrínseco cujo efeito opõe-se ao efeito paramagnético, os valores das susceptibilidades determinadas experi-

*e-mail: jaisa@quimica.ufpr.br mentalmente devem receber correções que contrabalancem a contribuição diamagnética inerente à natureza do material analisado.

Esta nota técnica apresenta um programa desenvolvido para o cálculo: (i) da contribuição diamagnética para a susceptibilidade de amostras de composição elementar conhecida, (ii) dos valores corrigidos da susceptibilidade magnética molar, considerando os seus componentes paramagnético e diamagnético e (iii) do momento magnético efetivo das substâncias de interesse. O programa emprega os conceitos de aditividade e de "transferability" (possibilidade de transferência) das contribuições atômicas ou de grupos no cálculo da susceptibilidade magnética total da amostra ${ }^{11}$. Ele foi concebido, nesta primeira versão, para usuários do método de Gouy ${ }^{10}$.

\section{TEORIA E DEFINIÇÕES}

As propriedades magnéticas de complexos de metais do bloco $d$ são freqüentemente expressas pelo momento magnético efetivo $\left(\mu_{\mathrm{ef}}\right)$ da amostra, uma vez que esta grandeza tem magnitudes mais convenientes que as da susceptibilidade magnética. Em sistemas "spinonly", ou seja, em que a contribuição orbital para a susceptibilidade é minimizada ou eliminada pelo efeito do campo ligante, o valor de $\mu_{\text {ef }}$ indica o número de elétrons desemparelhados, a força do campo ligante e a existência de interações antiferromagnéticas e ferromagnéticas entre centros metálicos. Para este cálculo, o programa MagnetoV1 utiliza a equação $\mu_{e f}=2,828 \sqrt{\chi_{M} T}$, onde $\chi_{\mathrm{M}}$ é a susceptibilidade magnética molar do complexo e T é a temperatura absoluta ${ }^{12}$.

As unidades empregadas pelo programa são do sistema cgs-emu, onde emu é uma abreviação de "electromagnetic units". Na realidade, embora o Sistema Internacional de Unidades (SI) seja hoje o mais utilizado na linguagem científica em geral, ele é pouco apropriado para as dimensões do magnetismo molecular ${ }^{8}$. A maioria dos pesquisadores deste campo utiliza o sistema cgs-emu e, no caso do programa Magneto-V1, a escolha deste sistema visou atender o maior número possível de usuários.

A susceptibilidade magnética $\chi$ é definida como:

$\chi=\mathbf{M} / \mathbf{H}$

onde $\mathbf{M}$ é a magnetização (ou momento magnético total) da amostra e $\mathbf{H}$, a força do campo magnético aplicado. Se a amostra é magneti- 
camente isotrópica, $\chi$ torna-se um escalar. A susceptibilidade magnética no volume total da amostra $\left(\chi_{\mathrm{v}}\right)$ é uma grandeza adimensional, mas é tradicionalmente expressa em emu $\cdot \mathrm{cm}^{-3}$. Portanto, emu tem dimensão de $\mathrm{cm}^{3}$. A susceptibilidade magnética por unidade de massa $\left(\chi_{\mathrm{g}}\right)$, expressa em emu.g ${ }^{-1}$, é calculada pela relação entre $\chi_{\mathrm{v}}$ e a densidade da amostra. Finalmente, a susceptibilidade magnética molar $\left(\chi_{\mathrm{M}}\right)$, expressa em emu $\cdot \mathrm{mol}^{-1}$, é obtida pela multiplicação de $\chi_{\mathrm{g}}$ pela massa molar do composto em análise ${ }^{10}$.

O programa Magneto-V1 permite a realização de cálculos com dados de amostras sólidas, líquidas ou em solução. Nas medidas em solução, determina-se $\chi_{\mathrm{v}(\mathrm{s})}$ (susceptibilidade magnética no volume total da solução) que deve ser transformado em $\chi_{\mathrm{s}}\left(\chi_{\mathrm{s}}\right.$ é o $\chi_{\mathrm{g}}$ da solução). A partir de $\chi_{\mathrm{s}}$ calcula-se o $\chi_{\mathrm{g}}$ do soluto (Equação 2) e daí o seu $\chi_{\mathrm{M}}{ }^{13}$.

$\chi_{S}=\left(\frac{m_{1}}{m_{1}+m_{0}}\right) \chi_{g}+\left(\frac{m_{0}}{m_{1}+m_{0}}\right) \chi_{0}$

Nesta equação, $m_{1}$ corresponde à massa do soluto e $m_{0}$ à massa do solvente. $\chi_{0}$ é a susceptibilidade magnética por unidade de massa do solvente $\left(\chi_{\mathrm{g}}\right.$ do solvente); seu valor está disponível em tabelas ${ }^{14}$ ou pode ser determinado experimentalmente ${ }^{15}$. O programa contém esses dados para os solventes de uso mais comum. Para amostras sólidas o cálculo é mais simples, pois o equipamento fornece $\chi_{\mathrm{v}}$ para o sólido, que é então convertido, pelo programa, em $\chi_{\mathrm{g}}$ e $\chi_{\mathrm{M}}{ }^{13}$.

\section{CORREÇÕES DIAMAGNÉTICAS}

O diamagnetismo tem sua origem na interação de elétrons pareados com o campo magnético e é uma propriedade de toda a matéria, pois mesmo materiais paramagnéticos possuem níveis eletrônicos internos preenchidos. A teoria do diamagnetismo vem sendo desenvolvida há várias décadas e, para algumas moléculas pequenas, cálculos ab initio da susceptibilidade diamagnética molar têm gerado resultados razoáveis ${ }^{8}$.

Susceptibilidades diamagnéticas são independentes da força do campo e da temperatura e geram uma contribuição negativa (tipicamente de $-1 \times 10^{-7} \mathrm{a}-1 \times 10^{-6} \mathrm{emu}^{-\mathrm{g}^{-1}}$ ) para a susceptibilidade magnética total do material em estudo ${ }^{10}$. Para compostos paramagnéticos de massa molecular baixa ou complexos metálicos polinucleares com ligantes leves, a contribuição diamagnética é geralmente pequena em relação ao componente paramagnético da susceptibilidade, podendo inclusive tornar-se desprezível em temperaturas baixas. No entanto, em sistemas de massa molecular elevada com poucos centros paramagnéticos, como os constituídos por metaloproteínas ou materiais híbridos orgânico-inorgânicos, ou em estudos em solução (especialmente soluções de interesse biológico), a determinação precisa da correção diamagnética é crucial. Nesses casos, a contribuição paramagnética (dependente da temperatura) pode ser apenas uma parte muito pequena do sinal total registrado, e o emprego de magnetômetros de alta sensibilidade pode tornar-se absolutamente necessário ${ }^{8,16,17}$. Para metaloproteínas, esta determinação pode envolver o estudo independente da susceptibilidade diamagnética da apoproteína, ou seja, do agregado de cadeias polipeptídicas desprovidas dos íons metálicos paramagnéticos, ou mesmo de derivados da proteína em que esses íons tenham sido substituídos por íons diamagnéticos ${ }^{17}$. Outra alternativa, empregada tanto para macromoléculas quanto para sistemas de menor massa molecular, consiste em extrair matematicamente a contribuição diamagnética, independente da temperatura, de um sinal dependente da temperatura ${ }^{8,18}$.

Em estudos do comportamento magnético de complexos metálicos mono- ou oligonucleares, como por exemplo compostos macrocíclicos ou metalocenos, a susceptibilidade diamagnética mo$\operatorname{lar}\left({ }^{\mathrm{D}} \chi_{\mathrm{M}}\right)$ também pode ser determinada experimentalmente pela me- dida de $\chi_{\mathrm{M}}$ para um análogo diamagnético, ou mesmo desmetalado, do composto. Quando isso não é possível, ou quando apenas uma estimativa de ${ }^{\mathrm{D}} \chi_{\mathrm{M}}$ é suficiente, pode-se explorar o princípio da aditividade das susceptibilidades diamagnéticas dos átomos dos ligantes, dos contra-íons e dos próprios metais nos compostos de interesse, através da utilização das constantes de Pascal ${ }^{8,11,12,19}$. Modelos teóricos recentes têm tido sucesso em justificar esta aditividade, demonstrada inicialmente por Henrichsen em 1888 e conhecida posteriormente como Regra de Pascal ${ }^{19}$, e em verificar a sua aplicabilidade $^{11,20}$. No entanto, deve-se enfatizar que o método, embora muito utilizado, pode levar a erros consideráveis em alguns sistemas $^{21}$, especialmente quando radicais orgânicos estão envolvidos ${ }^{18}$ ou em moléculas orgânicas em que átomos de hidrogênio ligados a um único centro de carbono são seqüencialmente substituídos por átomos de flúor ${ }^{22}$.

A partir de uma extensa tabulação empírica, Pascal observou que as susceptibilidades diamagnéticas molares $\left({ }^{\mathrm{D}} \chi_{\mathrm{M}}\right)$ podem ser calculadas pela equação

${ }^{\mathrm{D}} \chi_{\mathrm{M}}=\left(\Sigma \mathrm{n}_{\mathrm{A}} \chi_{\mathrm{A}}\right)+(\Sigma \lambda)$

onde $\mathrm{n}_{\mathrm{A}}$ corresponde ao número de átomos de cada elemento nos diferentes ambientes químicos da molécula, $\chi_{\mathrm{A}}$ é a susceptibilidade diamagnética molar de cada um desses átomos, e $\lambda$ é uma correção constitutiva, que depende da natureza das ligações químicas na molécula ${ }^{10}$. O valor de ${ }^{\mathrm{D}} \chi_{\mathrm{M}}$, calculado a partir da Equação (3) e dos valores de $\chi_{\mathrm{A}}$ e de $\lambda$ tabulados por Pascal, é usado para corrigir a susceptibilidade determinada experimentalmente ( ${ }^{\text {EXP }} \chi_{\mathrm{M}}$ ), gerando o $\chi_{\mathrm{M}}$ da amostra paramagnética:

$\chi_{\mathrm{M}}($ corrigido $)=\left({ }^{\mathrm{EXP}} \chi_{\mathrm{M}}\right)-\left({ }^{\mathrm{D}} \chi_{\mathrm{M}}\right)$

Como o valor da contribuição diamagnética $\left({ }^{\mathrm{D}} \chi_{\mathrm{M}}\right)$ é negativo, o cálculo expresso pela Equação (4) é, na realidade, uma adição.

No programa magneto-V1, o usuário indica o número de átomos e/ou grupamentos cujas contribuições diamagnéticas devem ser consideradas para cada amostra. O cálculo do valor corrigido do $\chi_{\mathrm{M}}$ da amostra é feito automaticamente.

Como mencionado anteriormente, o método das constantes de Pascal tem precisão apenas moderada, uma vez que as contribuições atômicas $\left(\chi_{A}\right)$ podem mudar de um composto para outro, dependendo do ambiente químico do átomo ou grupo de átomos considerado. De fato, para certos elementos, como o flúor, parece impossível propor um valor de contribuição diamagnética que realmente independa do restante do arcabouço molecular ${ }^{22}$. No entanto, discrepâncias deste tipo não invalidam os métodos aditivos ${ }^{8}$, embora a sua ocorrência não possa ser negligenciada. Em muitos casos, especialmente os que envolvem compostos paramagnéticos de massa molecular baixa e nas aplicações em que o cálculo do diamagnetismo não constitui a essência do experimento, as constantes de Pascal têm sido freqüentemente utilizadas, com resultados aceitáveis, na estimativa das contribuições diamagnéticas para a susceptibilidade magnética das substâncias em estudo.

\section{PARTE EXPERIMENTAL}

O programa Magneto-V1 foi desenvolvido na linguagem Perl 5.0 ("Practical Extraction and Report Language") ${ }^{23,24}$ com módulo CGI.pm 3.05 ("Common Gateway Interface") ${ }^{25}$ e está hospedado no servidor Unix do Departamento de Química da UFPR. O serviço está disponível para utilização "on-line" através do endereço eletrônico "http://www.quimica.ufpr.br/cgi-bin/mag/mag.cgi”. O acesso pode ser feito pela maioria dos navegadores de internet ("browsers"), 
tais como o "Internet Explorer", "Netscape", "Mozilla", "Opera", "Konqueror", entre outros, que suportem a versão 4.0 da linguagem HTML ("HyperText Markup Language") 26. Cópias do programa podem ser fornecidas gratuitamente aos interessados que as solicitarem aos autores.

As análises magnetoquímicas mencionadas nesta nota foram realizadas numa balança Johnson-Matthey MK II, disponível no Laboratório de Química Bioinorgânica da UFPR. Neste equipamento, as medidas são realizadas por uma modificação do método de Gouy ${ }^{13}$. A técnica modificada permite o emprego de quantidades pequenas de amostra, seja no estado sólido, em líquidos puros, ou em soluções.

\section{APRESENTAÇÃO DO PROGRAMA}

O programa é apresentado ao usuário na forma de quatro telas: duas possuem campos que devem ser preenchidos de acordo com a especificidade da amostra e duas apresentam os resultados.

Na tela inicial do programa são introduzidos os dados gerais da amostra e da análise (Figura 1), que são então usados para formatar as telas seguintes. Deve-se informar inicialmente se a análise foi realizada no sólido pulverizado ou em solução, pois o cálculo é diferenciado para os dois tipos de amostra. Além disso, deve-se selecionar a fase gasosa e a temperatura da realização do experimento. Dados de até 30 medidas podem ser inseridos e são utilizados para o cálculo dos valores médios das grandezas magnetoquímicas. Caso o usuário não disponha de dados experimentais no formato de entrada do programa e queira apenas calcular a correção diamagnética molar para a susceptibilidade de um determinado composto, deve informar "zero" no campo "número de medidas" e prosseguir com o cálculo. A informação sobre a fórmula molecular ou mínima solicitada na tela inicial (Figura 1) é utilizada apenas para identificar a análise, ou seja, não é empregada nos cálculos. Já a massa molar proposta para a substância paramagnética, que por sua vez é essencial aos cálculos, deve ser informada em tela posterior. A temperatura do experimento deve ser expressa em Kelvin.

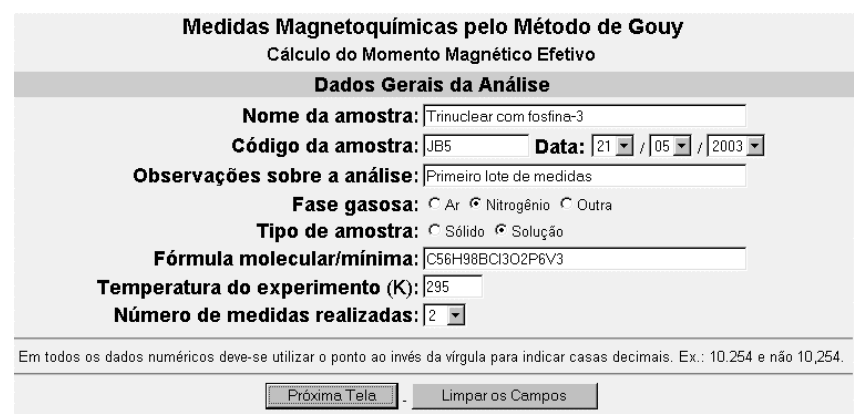

Figura 1. Tela inicial do programa Magneto-V1

A utilização do programa será exemplificada nas Figuras 1-5 com dados experimentais de uma solução do complexo trinuclear de vanádio(II) $\left[\mathrm{V}_{3} \mathrm{Cl}_{3}(\mathrm{OMe})_{2}(\text { depe })_{3}\right]\left(\mathrm{BPh}_{4}\right)$, depe = bis(difenilfosfino)etano, na concentração de $1,41 \times 10^{-2} \mathrm{~mol} \mathrm{~L}^{-1} \mathrm{em}$ tetraidrofurano (Figura 2) ${ }^{27}$. Este composto, descrito pela primeira vez por um dos autores desta nota, foi escolhido por conter átomos de sete elementos químicos distintos, além de possuir ligantes neutros e carregados e o contra-íon, o que permite a utilização de seções diferentes das tabelas de Pascal. O material apresenta comportamento antiferromagnético no estado sólido e em solução ${ }^{27}$.

$\mathrm{Na}$ segunda tela do programa (Figuras 3 e 4 ) devem ser informados dados referentes à amostra e às medidas, como as massas do soluto e do solvente, o nome do solvente utilizado, o número de
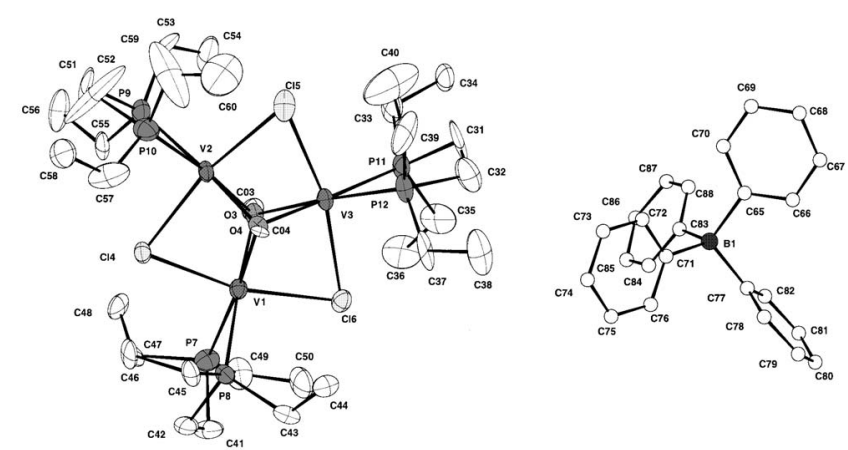

Figura 2. Diagrama ORTEP-3 para $\left[\mathrm{V}_{3} \mathrm{Cl}_{3}(\mathrm{OMe})_{2}(\text { depe })_{3}\right]\left(\mathrm{BPh} \mathrm{h}_{4}\right)$

centros paramagnéticos e a massa molar proposta para o composto. As duas colunas seguintes devem ser preenchidas com os valores medidos da susceptibilidade magnética do tubo vazio e do tubo com a solução-amostra. O número de medidas nessas duas colunas não precisa ser coincidente.

Na seção "Uso das Constantes de Pascal", o programa calcula as correções referentes aos tipos de ligação e de grupamentos presentes na molécula (constitutivas) e ao somatório das susceptibilidades diamagnéticas atômicas e iônicas. Para tanto, o usuário deve informar o número de ocorrências em cada campo, onde aplicável (Figura 4).

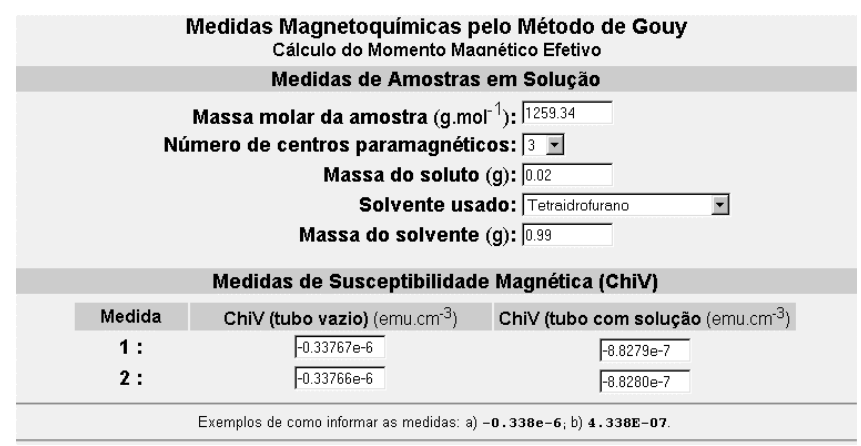

Figura 3. Tela na qual são introduzidos os dados específicos da análise

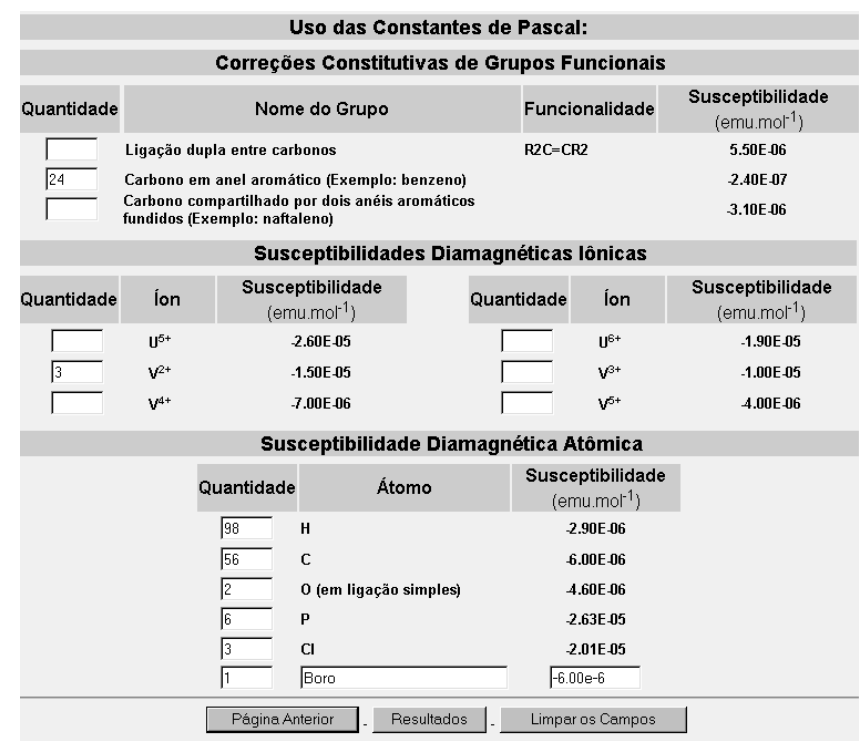

Figura 4. Tela com informações sobre as correções diamagnéticas a serem calculadas a partir das constantes de Pascal 
Em todas as subseções foram deixados campos em branco que permitem a introdução de dados novos. A Figura 4 mostra, por exemplo, a inserção do átomo de boro, com um $\chi_{\mathrm{A}}$ de $-6,00 \times 10^{-6} \mathrm{emu} \cdot \mathrm{mol}^{-1}$. $\mathrm{Na}$ ausência completa de informação sobre um elemento químico, pode-se utilizar, como aproximação, o valor de $\chi_{\mathrm{A}}$ disponível para outro elemento, desde que este possua um número similar de elétrons.

Na tela de resultados (Figura 5) são apresentados os valores calculados das susceptibilidades $\chi_{\mathrm{g}}$ e $\chi_{\mathrm{M}}$ (este último já incorporando as correções diamagnéticas) e do momento magnético efetivo total. Se a amostra possuir nuclearidade maior que um, será informado o momento magnético por centro metálico. Caso o usuário queira ter acesso a um relatório mais detalhado, deve selecionar a opção "Detalhe dos Cálculos", que o levará a uma tela com todos os dados informados pelo usuário, a lista completa das correções aplicadas, os valores das susceptibilidades corrigidas e do momento magnético efetivo. Para o mesmo conjunto de dados experimentais, várias formulações alternativas podem ser testadas rapidamente para a amostra, através do botão "Página Anterior".

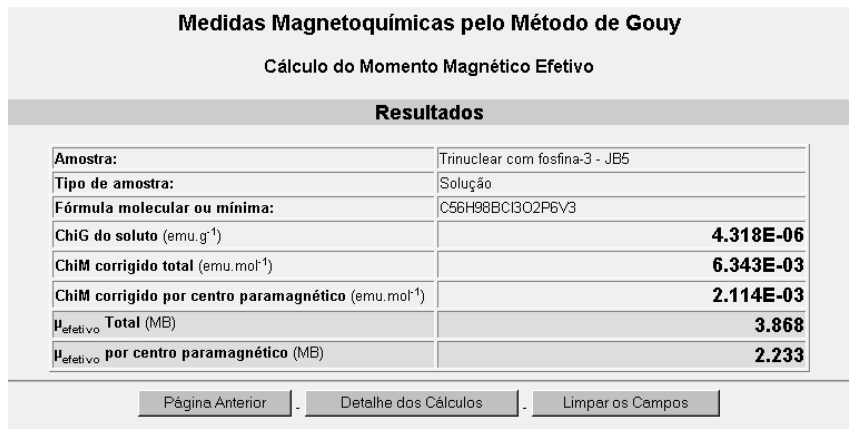

Figura 5. Tela de resultados do programa Magneto-V1

A interação dos usuários com os autores é bem-vinda, pois poderá contribuir para o desenvolvimento de novas versões do programa, mais adequadas às necessidades dos usuários. Os autores agradecem qualquer contribuição nesse sentido, especialmente aquelas que venham a completar as tabelas de dados e ampliar a aplicabilidade do programa.

\section{CONCLUSÃO}

O programa Magneto-V1 permite que os resultados de medidas magnetoquímicas sejam confrontados rapidamente com diversas formulações propostas para uma substância de interesse. Trata-se de uma ferramenta útil na caracterização estrutural e eletrônica de novos complexos paramagnéticos.

\section{AGRADECIMENTOS}

Os autores agradecem aos professores E. L. de Sá e C. Carvalho pela hospedagem do Programa Magneto-V1 no servidor do Departamento de Química da UFPR; ao Dr. P. B. Hitchcock (Department of Chemistry, University of Sussex, UK) pela representação ORTEP da Figura 2; à Universidade Federal do Paraná, ao PRONEX (contrato $\mathrm{n}^{\mathrm{o}}$ 41.96.0884.00), à FINEP (Financiadora de Estudos e Projetos), ao CNPq (Conselho Nacional de Desenvolvimento Científico e Tecnológico) e à Fundação Araucária, pelo apoio financeiro. G. G. Nunes e G. R. Friedermann agradecem à Coordenação de Aperfeiçoamento de Pessoal de Nível Superior (CAPES) pela concessão de bolsas de doutorado.

\section{REFERÊNCIAS}

1. Carneiro, A. A. O.; Touso, A. T.; Baffa, O.; Quim. Nova 2003, 26, 952; Spencer, B.; Itami, Y.; Sone, K.; J. Chem. Educ. 2002, 79, 1002; de Berg, K. C.; Chapman, K. J.; J. Chem. Educ. 2001, 78, 670; Matsuyama, Y.; Yasuoka, T.; Mitsuzawa, S.; Sasaki, T.; J. Chem. Educ. 1997, 74, 943; Piguet, C.; J. Chem. Educ. 1997, 74, 815; Shimada, H.; Yasuoka, T.; Mitsuzawa, S.; J. Chem. Educ. 1990, 67, 63; Zare, R. N.; J. Chem. Educ. 1988, 65, 277; Quickenden, T. I.; Marshall, R. C. G.; J. Chem. Educ. 1972, 49, 114.

2. Cornia, A.; Gatteschi, D.; Sessoli, R.; Coord. Chem. Rev. 2001, 219, 573; Carlin, R. L.; Coord. Chem. Rev. 1987, 79, 215; Cairns, C. J.; Busch, D. H.; Coord. Chem. Rev. 1986, 69, 1; Carlin, R. L.; Acc. Chem. Res. 1976, 9, 67; Reis, D. M.; Nunes, G. G.; Sá, E. L.; Friedermann, G. R.; Mangrich, A. S.; Evans, D. J.; Hitchcock, P. B.; Leigh, G. J.; Soares, J. F.; New J. Chem. 2004, 28, 1168; Vaz, M. G. F.; Knobel, M.; Speziali, N. L.; Moreira, A. M.; Alcantara, A. F. C.; Stumpf, H. O.; J. Braz. Chem. Soc. 2002, 13, 183; Neves, A.; Rossi, L. M.; Bortolozzi, A. J.; Mangrich, A. S.; Haase, W.; Werner, R.; J. Braz. Chem. Soc. 2001, 12, 747; de Lima, R. L.; Teixeira, L. R. D.; Carneiro, T. M. G.; Beraldo, H.; J. Braz. Chem. Soc. 1999, 10, 184.

3. Verdaguer, M.; Polyhedron 2001, 20, 1115; Filippou, A. C.; Schneider, S.; Schnakenburg, G.; Inorg. Chem. 2003, 42, 6974; Gobel, A.; Leibeling, G.; Rudolph, M.; Imhof, W.; Organometallics 2003, 22, 759; Nunes, G. G.; Reis, D. M.; Camargo, P. H. C.; Hitchcock, P. B.; Horner, M.; Matos, R. M.; Mangrich, A. S.; Sá, E. L.; Leigh, G. J.; Soares, J. F.; J. Braz. Chem. Soc. 2003, 14, 922; Canada-Vilalta, C.; Rumberger, E.; Brechin, E. K.; Wernsdorfer, W.; Folting, K.; Davidson, E. R.; Hendrickson, D. N.; Christou, G.; J. Chem. Soc., Dalton Trans. 2002, 4005; Nunes, G. G.; Reis, D. M.; Amorim, P. T.; Sá, E. L.; Mangrich, A. S.; Evans, D. J.; Hitchcock, P. B.; Leigh, G. J.; Nunes, F. S.; Soares, J. F.; New J. Chem. 2002, 26, 519.

4. Jiles, D. C.; Acta Mater. 2003, 51, 5907; Kahn, O.; Acc. Chem. Res. 2000, 33,647 .

5. Marvaud, V.; Herrera, J. M.; Barilero, T.; Tuyeras, F.; Garde, R.; Scuiller, A.; Decroix, C.; Cantuel, M.; Desplanches, C.; Monatsh. Chem. 2003, 134, 149; Letard, J. F.; Chastanet, G.; Nguyen, O.; Marcen, S.; Marchivie, M.; Guionneau, P.; Chasseau, D.; Gutlich, P.; Monatsh. Chem. 2003, 134, 165; Goujon, A.; Varret, F.; Escax, V.; Bleuzen, A.; Verdaguer, M.; Polyhedron 2001, 20, 1339; Gutlich, P.; Garcia, Y.; Woike, T. Coord. Chem. Rev. 2001, $219,839$.

6. Pereira, C. L. M.; Pedroso, E. F.; Stumpf, H. O.; Novak, M. A.; Ricard, L.; Ruiz-Garcia, R.; Riviere, E.; Journaux, Y.; Angew. Chem., Int. Ed. 2004, 43, 955; Mikhaylova, M.; Kim, D. K.; Bobrysheva, N.; Osmolowsky, M.; Semenov, V.; Tsakalakos, T.; Muhammed, M.; Langmuir 2004, 20, 2472; Skumryev, V.; Stoyanov, S.; Zhang, Y.; Hadjipanayis, G.; Givord, D.; Nogues, J.; Nature 2003, 423, 850; Marchetti, S. G.; Mercader, R. C.; Hyperfine Interact. 2003, 148, 275; Cowburn, R. P.; J. Appl. Phys. 2003, 93, 9310; Gibbs, M. R. J.; Curr. Opin. Solid St. M. 2003, 7, 83; Carreno, N. L. V.; Leite, E. R.; Santos, L. P. S.; Lisboa, P. N.; Longo, E.; Araújo, G. C. L.; Barison, A.; Ferreira, A. G.; Valentini, A.; Probst, L. F. D.; Quim. Nova 2002, 25, 935; Binns, C.; J. Nanosci. Nanotechnol. 2001, 1, 243; Wiesendanger, R.; Curr. Opin. Solid St. M. 1999, 4, 435.

7. Rosen, A. D.; Cell Biochem. Biophys. 2003, 39, 163; Mapps, D. J.; Sens. Actuators, A 2003, 106, 321; Coey, J. M. D.; Kobayashi, K.; Uchikawa, Y.; IEEE Trans. Magn. 2003, 39, 3378; J. Magn. Magn. Mater. 2001, 226, 2107; Kahn, O.; Martinez, C.J.; Science 1998, 279, 44; Donaire, A.; Salgado, J.; Moratal, J. M.; Biochem. 1998, 37, 8659; Mulay, L. N.; Mulay I. L.; Anal. Chem. 1980, 52, 199R.

8. Kahn, O.; Molecular Magnetism, VCH: New York, 1993.

9. Bencini, A.; Carbonera, C.; Totti, F.; Chem.-Eur. J. 2004, 10, 1472; Bencini, A.; Dei, A.; Sangregorio, C.; Totti, F.; Vaz, M. G. F.; Inorg. Chem. 2003, 42, 8065; Autschbach, J.; Ziegler, T.; Coord. Chem. Rev. 2003, 238, 83.

10. O'Connor, C. J.; ACS Symp. Ser. 1996, 644, 44; Drago, R. S.; Physical methods for chemists, $2^{\mathrm{a}}$ ed., Saunders College: Philadelphia, 1992; O'Connor, C. J.; Prog. Inorg. Chem. 1982, 29, 203.

11. Bader, R. F. W.; Keith, T. A.; J. Chem. Phys. 1993, 99, 3683; CortésGuzmán, F.; Bader, R. F. W.; Chem. Phys. Lett. 2003, 379, 183.

12. Carlin, R. L.; Magnetochemistry, Springer-Verlag: Berlin, 1986.

13. Woolcock, J.; Zafar, A.; J. Chem. Educ. 1982, 69, A177; Teweldemedhin, Z. S.; Fuller, R. L.; Greenblatt, M.; J. Chem. Educ. 1996, 73, 906.

14. Becker, E. D.; High Resolution Nuclear Magnetic Resonance, $2^{\mathrm{a}}$ ed., Academic Press: New York, 1980.

15. Mizuno, K.; Ochi, T.; Shimada, S.; Nishimura, Y.; Maeda, S.; Koga, Y.; Phys. Chem. Chem. Phys. 1999, 1, 133; Sandifer, M. E.; Zhao, M.; Kim, S. H.; Scherson, D. A.; Anal. Chem. 1993, 65, 2093; Bothnerby, A. A.; Gayathri, C.; Vanzijl, P. C. M.; MacLean, C.; J. Magn. Reson. 1984, 56, 456; Sueoka, K; Bull. Chem. Soc. Jpn. 1991, 64, 916. 
16. Donaire, A.; Jimenez, B.; Moratal, J. M.; Hall, J. F.; Hasnain, S. S.; Biochemistry 2001, 40, 837; Bertini, I.; Rosato, A.; Turano, P.; Pure Appl. Chem. 1999, 71, 1717.

17. Morgenstern-Badarau, I.; Cocco, D.; Desideri, A., Rotilio, G.; Jordanov, J. Dupre, N.; J. Am. Chem. Soc. 1986, 108, 300.

18. Sugano, T.; Polyhedron 2001, 20, 1285.

19. Henrichsen, G.; Wied. Ann. 1888, 34, 186; Pascal, P.; Anal. Chem. Phys. 1910, 19, 5 .

20. Astrand, P. O.; Mikkelsen, K. V.; Mag. Reson. Chem. 1998, 36, 92; Ruud, K.; Skaane, H.; Helgaker, T.; Bak, K. L.; Jorgensen, P.; J. Am. Chem. Soc. 1994, 116, 10135.

21. Vaz, M. G. F.; Pinheiro, L. M. M.; Stumpf, H. O.; Alcantara, A. F. C.; Golhen, S.; Ouahab, L.; Cador, O.; Mathoniere, C.; Kahn, O.; Chem.- Eur. J. 1999, 5, 1486 .

22. Ruud, K.; Astrand, P. O.; Taylor, P. R.; J. Phys. Chem. A 2001, 105, 9926.

23. Wall, L; Christiansen, T.; Orwant, J.; Programming Perl, $3^{\mathrm{a}}$ ed., Oreilly: Cambridge MA, 2000; http://www.perl.org/, acessada em Junho 2004.
24. Brown, M. C.; Active Perl - Guia do Programador, Ciência Moderna: Rio de Janeiro, 2001.

25. Stein, L. D.; CGI.pm 3.05: Simple Common Gateway Interface Class, Cold Spring Harbor Laboratory, Cold Spring Harbor: New York, 2004; http:// stein.cshl.org/, acessada em Junho 2004; http://search.cpan.org/ lds/ CGI.pm-3.05/, acessada em Junho 2004; http://search.cpan.org/ search?query=cgi\&mode=all, acessada em Junho 2004.

26. Raggett, D.; Le Hors, A.; Jacobs, I.; HTML 4.01: HyperText Markup Language, Rutherford Appleton Laboratory, Oxfordshire, UK, 1999; http:/ /www.w3.org/TR/html401/, acessada em Junho 2004.

27. Hitchcock P. B.; Hughes, D. L.; Larkworthy, L. F.; Leigh, G. J.; Marmion, C. J.; Sanders, J. R.; Smith, G. W.; de Souza, J. S.; J. Chem. Soc., Dalton Trans. 1997, 1127.

28. Farrugia, L. J.; J. Appl. Crystallogr. 1999, 32, 837; Farrugia, L. J.; J. Appl. Crystallogr. 1997, 30, 565. 